Check for updates

Cite this: J. Mater. Chem. C, 2018, 6, 5046

Received 1st February 2018,

Accepted 22nd March 2018

DOI: $10.1039 / \mathrm{c} 8 \mathrm{tc0} 0564 \mathrm{~h}$

rsc.li/materials-c

\section{Controlling the charge transfer flow at the graphene/pyrene-nitrilotriacetic acid interface $\dagger$}

\author{
Silvio Osella, (D)*a Matgorzata Kiliszek, ${ }^{b}$ Ersan Harputlu, ${ }^{c}$ Cumhur G. Unlu, ${ }^{d}$ \\ Kasim Ocakoglu, ${ }^{\text {ce }}$ Joanna Kargul ${ }^{* b}$ and Bartosz Trzaskowski (D) ${ }^{a}$
}

\section{Introduction}

Immobilisation of redox active proteins on various types of inorganic and carbon-based electrode surface has been important in various biotechnological applications, such as biosensors, biofuel cells, solar-to-fuel devices and biomolecular nanoelectronics. ${ }^{1-3}$ The state-of-the-art in these fields is based on developing optimal interfaces for highly oriented biomolecular conjugation of the electroactive proteins that would assure efficient direct electron transport (DET) while preserving the full catalytic activity of the inbound biological modules. Anchoring of a redox active enzyme to the appropriately functionalised solid surface via a genetically engineered affinity tag, such as a $\mathrm{His}_{6}$-tag, opens the possibility of advanced biophysical studies even at the single molecule level. ${ }^{4-8}$

In order to improve the electronic communication between the enzyme and the electrode surface, new nanoscale materials and metallic nanoparticles have been incorporated into the bioelectrode structure. Of all the electrode materials, graphene

\footnotetext{
${ }^{a}$ Chemical and Biological Systems Simulation Lab, Centre of New Technologies, University of Warsaw, Banacha 2C, 02-097 Warsaw, Poland.

E-mail: s.osella@cent.uw.edu.pl

${ }^{b}$ Solar Fuels Lab, Centre of New Technologies, University of Warsaw, Banacha $2 C$, 02-097 Warsaw, Poland. E-mail: j.kargul@cent.uw.edu.pl

${ }^{c}$ Advanced Technology Research \& Application Centre, Mersin University, Ciftlikkoy Campus, TR33343, Yenisehir, Mersin, Turkey

${ }^{d}$ Department of Biomedical Engineering, Pamukkale University, TR-20070 Denizli, Turkey

${ }^{e}$ Department of Energy Systems Engineering, Mersin University,

Tarsus Faculty of Technology, 33480 Mersin, Turkey

$\dagger$ Electronic supplementary information (ESI) available: Geometrical analysis of the optimized structures, analysis of a different conformation of the G/pyrNTA interface, and photochronoamperometric analysis. See DOI: 10.1039/c8tc00564h
}

and its derivatives have provided a nanomaterial of choice for nanoengineering of bioelectrodes and bionanoelectronic devices due their remarkable physical properties, such as the large surface area of the two-dimensional $\mathrm{sp}^{2}$-hybridised honeycomb single layer carbon lattice, exceptional mechanical strength, transparency, elasticity, flatness, as well as excellent electrical and heat conductivity and amenability to attach functional groups. ${ }^{9-18}$ The atomic thickness of the graphene lattice facilitates access to the immobilised enzymatic active sites, thus enhancing electronic communication with redox active proteins and regeneration of their redox centres through direct or mediated electron transfer. ${ }^{19-22}$ In addition, the unique location of its Fermi level at the intersection between the top of the valence band and the bottom of the conduction band favours ambipolar charge transport, since the charge carrier density can be continuously tuned for electrons or holes, with the mobilities measured for exfoliated graphene exceeding $50000 \mathrm{~cm}^{2} \mathrm{~V}^{-1} \mathrm{~s}^{-1}$ under ambient conditions. ${ }^{9}$ Despite these astonishing properties, to be used in real devices, a band gap, even of the order of a few tenths of $\mathrm{eV}$, has to be opened. In addition, it is imperative to develop rational approaches for the improvement of DET between various modules of the graphene-based bioelectrodes in order to minimise the wasteful back reactions leading to the decrease of the electrode performance, such as charge recombination. There are multiple studies which show that very fast charge recombination is responsible for the inefficient photovoltaic conversion for a number of otherwise promising interfaces. ${ }^{23-25}$ Therefore, the precise definition of the electron transfer parameters within the conductive interface between the graphene surface and the redox centre of the biological module of the electrode aids the rational nanostructuring of such devices aimed at improvement of the power output. 
One of the most effective methods of preserving the remarkable conductive properties of the graphene is to form non-covalent functional structures with molecules containing common $\pi$-systems, such as pyrene or its derivatives to form a molecular interface between the catalytic modules and the graphene monolayer. ${ }^{26,27}$ With this approach, the $\pi$-conjugated structure of graphene, and thus its high charge mobility, is preserved, but the reversibility of the interaction can lead to the desorption of the molecules from the surface. The class of pyrene-derivative molecules has proven to be successful for stability, integration and preservation of the activity of redox active catalysts, such as the water-splitting $\mathrm{Cu}$ catalyst, ${ }^{28}$ and photoactive enzymes, such as photosystem I, following their immobilisation on graphenebased electrodes. ${ }^{29}$ Thus, the creation of a stable interface between graphene and a self-assembled monolayer (SAM) is of crucial importance to improve the DET in between the biological system and the metal electrode and to obtain a high performance device for biomolecular nanoelectronics.

In this work, we report a joint computational and electrochemical study on the properties of a well-defined SAM of pyrene derivative molecules and their ability to tune the electronic properties of graphene when the interface is introduced. The work function modification of graphene due to the presence of the SAM has been analysed by means of Density Functional Theory (DFT) calculations, and the results have been validated by chronoamperometric analysis. Both analyses show an increase in the work function of graphene due to the presence of the pyrene-nitrilotriacetic acid SAM, which, in turn, leads to charge transfer from the SAM to graphene when $\mathrm{Ni}^{2+}$ and imidazole coordination are present in the system, allowing for the fine control and tuning of the DET.

\section{Materials and methods}

\section{Quantum mechanical calculations}

A hexagonal 2D slab of monolayer graphene with lattice parameters $a=10.70 \AA$; $b=6.179 \AA, \alpha=120^{\circ}$ and a C-C distance of $1.43 \AA$ was manually built and optimized. One pyrene-nitrilotriacetic acid or pyrene-nitrilotriacetic acid- $\mathrm{Ni}^{2+}$-imidazole molecule was subsequently physisorbed on the graphene layer and a vacuum region of $30 \AA$ was introduced in the third dimension to avoid spurious electrostatic interactions between the replica (Fig. 1). Since the final system of the graphene/ pyrene self-assembled monolayer (hereafter dubbed G/SAM) is formed by more than 120 atoms, the computational cost of Density Functional Theory (DFT) becomes prohibitive, particularly when considering the Periodic Boundary Condition (PBC) to describe solid-state systems. For this reason, a tight-binding (TB) DFT method has been considered for the geometry optimization. ${ }^{30}$ In this approach, the wave function of a complex system is constructed as a superposition of the wave functions for isolated atoms located at the positions of the corresponding nuclei within the system of interest. This approach has been successfully used to describe graphene and its derivatives, achieving accuracies comparable to high $a b$ initio methods,

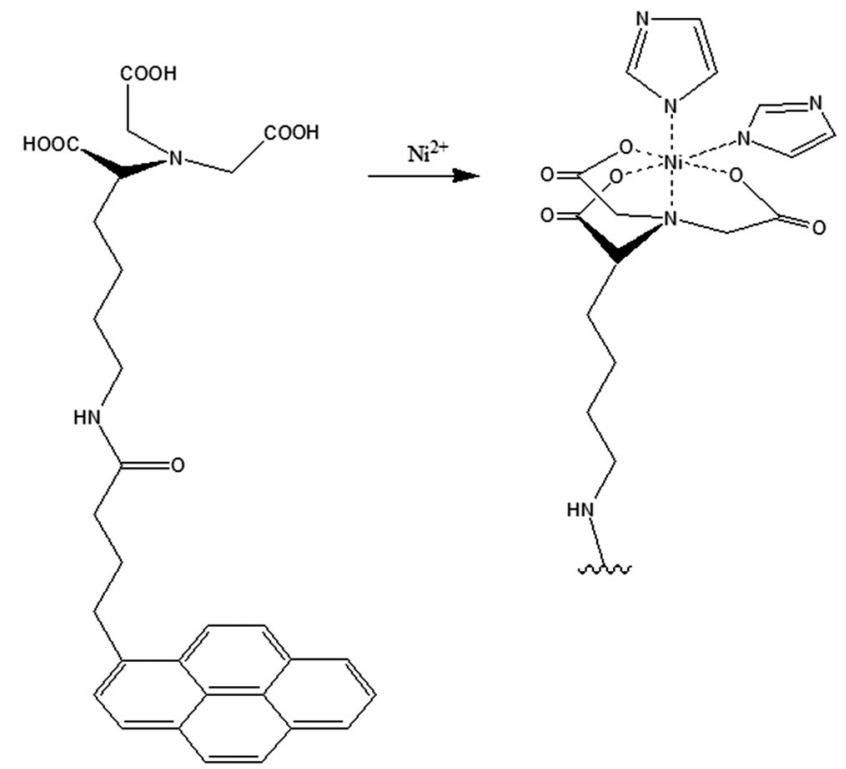

Fig. 1 Chemical structures of the SAM-forming molecules studied without the metal ion (pyrNTA, left) and with the presence of chelation (pyrNTA-Ni-IM, right).

with the advantage of being able to simulate medium-large systems (comprised of hundreds of atoms). ${ }^{31-33}$ The Coulomb interaction between the fluctuation of charge has been incorporated by the self-consistent redistribution of the Mulliken charges (SSC), ${ }^{34}$ to account for the long-range electrostatic and self-interaction contributions, enhancing the accuracy of the method in the description of electronic properties of solids. ${ }^{35}$ To account for an accurate description of non-covalent interactions, such as the one present in the current investigated system, empirical dispersion corrections as done in DFT to create DFT-D methods (i.e. Grimme's dispersion terms), considering the van der Waals dispersions (DFTB-D) ${ }^{36}$ to properly describe the $\pi-\pi$ nature of the interaction of molecules physisorbed on the graphene monolayer, have been introduced. The DFTB calculations have been performed with the DFTB+ software. $^{37}$

DFT periodic calculations have been performed on the optimized structures with the PWscf package of the Quantum Espresso suite of programs. ${ }^{38}$ Since van der Waals interactions play a major role in the stability of the G/SAM system, the PBE functional with the vdw-DF2 term for the exchange and correlation was used. ${ }^{39}$ Ultrasoft pseudopotentials with a cut-off of 50 and $200 \mathrm{Ry}$ for the expansion of the wave function and density were applied, respectively. In addition, the dipole correction for the electrostatic potential was taken into account to counterbalance the asymmetry in the electrostatic potential arising from the formation of the G/SAM interface. A $2 \times 2 \times 1$ $k$-sampling was used for the analysis of the Brillouin zone, at the $\Gamma$ point. We would like to stress here that all the analyses have been performed at the $\Gamma$ point. Test calculations on larger $k$-sampling of $4 \times 4 \times 1$ resulted in similar values of energy, thus ensuring the convergence of the calculations. The charge analysis was performed using the Bader algorithm. 
The obtained electronic density was used to plot the averaged electrostatic potential along the axis normal to the interface (i.e. $z$-axis). In this way, it was possible to directly estimate the work function shift by comparing the converged potential on the bare side and on the SAM-covered side of the surface. Two main contributions are responsible of the total shift in the work function $(\Delta \Phi)$ : the first arises from the dipole moment of the molecular backbone, named hereafter the molecular contribution $\left(\Delta \Phi_{\text {SAM }}\right)$ and the second from the interfacial electronic reorganization upon physisorption of the SAM on graphene, namely the charge transfer contribution $\left(\Delta \Phi_{\mathrm{CT}}\right)$ :

$$
\Delta \Phi=\Delta \Phi_{\mathrm{SAM}}+\Delta \Phi_{\mathrm{CT}}
$$

$\Delta \Phi_{\mathrm{SAM}}$ has been estimated here by computing the electrostatic potential profile across the molecules without graphene, while keeping the coordinates of the system frozen. The $\Delta \Phi_{\mathrm{CT}}$ contribution is then calculated by subtracting $\Delta \Phi_{\mathrm{SAM}}$ from $\Delta \Phi$. Only when a dipole moment is present in the SAM is the shift in the work function observed.

In the Helmholtz model, the molecular contribution $\Delta \Phi_{\mathrm{SAM}}$ is directly proportional to the molecular dipole along the axis normal to the surface, $\mu_{\perp}$, and inversely proportional to the surface area per adsorbed molecule A:

$$
\Delta \Phi_{\mathrm{SAM}}=e \mu_{\perp} / \varepsilon_{0} \mathrm{~A}
$$

with $\varepsilon_{0}$ as the vacuum permittivity. ${ }^{40,41}$ As a consequence, if the SAM dipole is pointing towards the metal surface the shift in the work function is positive, while if the dipole points away from the surface the shift in the work function is negative.

\section{Preparation of single layer graphene on an FTO substrate}

Single layer graphene (SLG) was produced on pure $\mathrm{Cu}$ foil which is used as a metallic catalyzer by chemical vapor deposition (CVD). The graphene layer was enlarged on the $\mathrm{Cu}$ foil and then transferred onto fluorine-doped tin oxide (FTO). To separate the $\mathrm{Cu}$ and graphene layers, a polymethyl methacrylate (PMMA) solution was overlaid homogeneously onto the graphene layer by spin coating to provide support for the transparent graphene layer. Subsequently, iron(III) nitrate solution was used to etch the Cu substrate. The PMMA-graphene layer was washed with deionized water and placed in $2 \% \mathrm{HCl}$ solution to remove metal contaminants. The graphene-PMMA layer was transferred to the FTO-coated glass surfaces. After this process, the PMMA/ graphene/FTO substrate was left in acetone to remove the PMMA layer. $^{42-46}$

\section{Functionalization of the graphene surface with pyrene-nitrilotriacetic acid (pyrNTA)}

The pyrNTA synthesis was performed as described in ref. 47 . For the modification of the graphene layer via $\pi-\pi$ stacking interactions, a solution of pyrNTA in $N, N$-dimethylformamide (DMF) was coated on the single layer graphene/fluorine-doped tin oxide (SLG/FTO) surface. After $1 \mathrm{~h}$ of incubation, the pyrNTA/SLG/FTO substrate was rinsed thoroughly with DMF, and then incubated with nickel(II) sulfate $\left(\mathrm{NiSO}_{4}\right)$ in Milli-Q water for $1 \mathrm{~h}$ to coordinate $\mathrm{Ni}^{2+}$ cations with the NTA-pyrene moiety on the SLG films. ${ }^{42,48}$ The elemental characterization of the functionalized electrodes was performed by energydispersive X-ray spectroscopy (EDX) using a field emission scanning electron microscope (FE-SEM, Zeiss/Supra 55) attached with an energy dispersive spectrometer.

\section{Electrochemical measurements}

A Versa STAT 3 (Princeton Applied Research, USA) potentiostat/ galvanostat connected with a KL 2500 LCD halogen white light source (Schott, Germany) was used for photoelectrochemical investigations. $\mathrm{An} \mathrm{Ag} / \mathrm{AgCl}(3 \mathrm{M} \mathrm{KCl})$ electrode and a platinum wire were used as the reference (REF) and counter (CE) electrodes, respectively. The Ni-NTA-pyrene/SLG coated on FTO glass (geometric surface area of $0.4185 \mathrm{~cm}^{2}$ ) was utilised as a working electrode (WE), with electrical contact provided through the copper tape with a conductive adhesive (6.4 mm width, 1181, $3 \mathrm{M}$ ). All electrochemical measurements were carried out in a custom-made Teflon cell filled with $5 \mathrm{mM}$ phosphate buffer $(\mathrm{pH} 7)$ as an electrolyte. The open circuit potential (OCP) was recorded in the dark and under illumination with light of $100 \mathrm{~mW} \mathrm{~cm} \mathrm{~cm}^{-2}$. The photochronoamperometric experiments were performed at different potentials ( $v s$. REF/OCP) for each electrode, with $30 \mathrm{~s}$ light ON/OFF periods. All electrochemical measurements were conducted under aerobic conditions at RT.

\section{Results and discussion}

\section{Theoretical calculation of the direct electron transfer at the graphene/SAM interface}

Two systems have been considered in the present study. The first system consists of a pyrene-nitrilotriacetic acid (pyrNTA) derivative physisorbed as an SAM on a graphene monolayer, while the second one includes the pyrNTA SAM system with $\mathrm{Ni}^{2+}$ cation coordination. In the construction of bioelectrodes and biosensors, the $\mathrm{Ni}^{2+}$-NTA moiety is used for the oriented immobilisation of (photo)electroactive proteins to capture the $\mathrm{His}_{6}$-tag genetically engineered within the structure of proteins of interest. To complete the octahedral coordination of the $\mathrm{Ni}^{2+}$ cation and to mimic the experimental setup of the $\mathrm{His}_{6}$-tag, two imidazole molecules have been added to the model (dubbed pyrNTA-Ni-IM, Fig. 1).

Both molecules were physisorbed on the graphene monolayer and after the optimization at the DFTB level of theory (more geometrical details are reported in the ESI, $\uparrow$ Table S1 and Fig. S1), a minimum distance of $2.9 \AA$ between pyrene and graphene was measured, with pyrene oriented on the graphene surface in a Bernal-stacking pattern. To preserve the neutral charge of the system, necessary for the PBC calculations, one of the carboxylic groups was protonated for systems with the $\mathrm{Ni}^{2+}$ cation. Interestingly, after the geometry optimization, the SAM assumes a 'sandwiched' conformation on the graphene surface, in which both the amidic group and the NTA moiety interact with the pyrene (see inset in Fig. 3). This can be rationalized by considering that the carboxylic groups of NTA tend to interact 
with the pyrene moiety to maximize the interaction energy. The adsorption energy of the G/SAM complex has been obtained as:

$$
E_{\mathrm{ads}}=E_{\mathrm{G} / \mathrm{SAM}}-\left(E_{\mathrm{G}}+E_{\mathrm{SAM}}\right)
$$

where the $E_{\mathrm{G}}$ and $E_{\mathrm{SAM}}$ are the contributions of the two components of the system calculated at the optimized geometry of the entire system. Adsorption energy values of -0.37 and $-0.22 \mathrm{eV}$ were found for the G/pyrNTA and G/pyrNTA-Ni-IM interfaces, respectively, ensuring the stability of both complexes.

The plane average potential profiles associated with the pyrNTA and pyrNTA-Ni-IM structures physisorbed on graphene, in their unit cell, are depicted in Fig. 2. The calculated work function for the modified graphene monolayer is equal to 5.11 and $6.01 \mathrm{eV}$ for G/pyrNTA and G/pyrNTA-Ni-IM, respectively. These results represent an increase by 0.42 and $1.29 \mathrm{eV}$ with respect to the bare graphene surface work function, with a
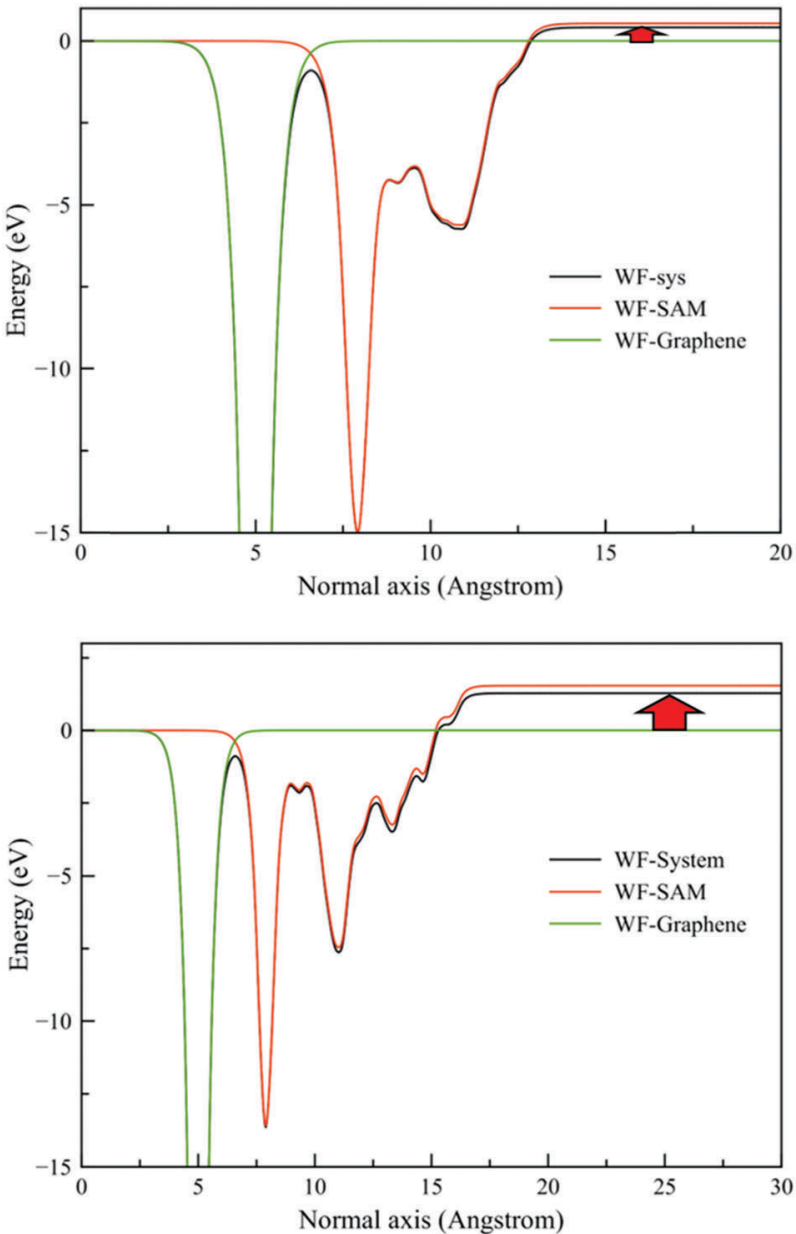

Fig. 2 Plane averaged potential of the G/pyrNTA (top) and G/pyrNTA-Ni-IM (bottom) interfaces. The displayed curves refer to the bare graphene monolayer (green), the graphene surface covered by SAM (black) and the free SAM layer (red). Going from left to right, we move from the graphene surface to the SAM contribution, away from the surface. For the sake of clarity, the vacuum region after the SAM is not shown. The red thick arrow indicates the total work function shift of the G/SAM interface. The difference between this shift and the SAM contribution corresponds to the charge transfer. computed value of $4.70 \mathrm{eV}$, in good agreement with experimental measurements of $4.6-4.8 \mathrm{eV} .{ }^{49}$ Interestingly, a slight change in conformation of pyrNTA does not change the overall picture of the WF shift (for more details, see the ESI $\dagger$ ).

To investigate the different contributions to the total work function shift $(\Delta \Phi)$, we analysed the two components separately in order to quantify the contribution from the charge transfer $\left(\Delta \Phi_{\mathrm{CT}}\right)$ versus the dipole supported by the molecular backbone $\left(\Delta \Phi_{\mathrm{SAM}}\right)$. The computed shifts arising from the free SAM are reported in Fig. 2 (red curve). For the pyrNTA system the contribution of the molecular dipole was found to be $0.54 \mathrm{eV}$, while when the $\mathrm{Ni}^{2+}$ cation and the two imidazole molecules were added, a stronger dipole of $1.55 \mathrm{eV}$ has been obtained. For both SAM structures, the charge transfer contribution (supramolecular effect) was found to be small, with values of -0.12 and $-0.26 \mathrm{eV}$ for pyrNTA and pyrNTA-Ni-IM SAMs, respectively. Thus, the total shift in the work function of the G/SAM interfaces is mainly driven by the molecular dipole of the SAM.

The molecular backbone contribution of the SAM to $\Delta \Phi$ arises from its dipole moment. For the pyrNTA structure without graphene, the value of the dipole moment component (in the unit cell) perpendicular to the graphene was found to be $-1.88 \mathrm{D}$, while the addition of the $\mathrm{Ni}^{2+}$ cation and two imidazole molecules changed it to $-5.42 \mathrm{D}$. The strong difference $(3.54 \mathrm{D})$ in dipole moment values due to the chelation of NTA with a $\mathrm{Ni}^{2+}$ cation, is the key to quantify the strong contribution of $\Delta \Phi_{\mathrm{SAM}}$ to the total work function. Depolarization effects strongly modify the molecular dipole moment of the interface when accounting for the formation of the interface with graphene. The total dipole is thus reduced by 22 and $17 \%$ for the G/pyrNTA and for G/pyrNTA$\mathrm{Ni}$-IM interfaces, leading to a final dipole per cell of -1.46 and $-4.51 \mathrm{D}$, respectively.

Interestingly, the charge transfer contribution $\Delta \Phi_{\mathrm{CT}}$, though relatively small, is not confined to the G/SAM interface (i.e. the interaction between graphene and pyrene) but evolves all the way to the end of the molecular backbone (Fig. 3). The difference between the two curves changes from zero to $0.14(-0.26) \mathrm{eV}$ moving away from the G/pyrNTA (G/pyrNTA-Ni-IM) interface. The plot illustrates that the charge transfer contribution is similar for the two systems when the graphene/pyrene interaction is considered, but it evolves in different ways (namely it is higher when the $\mathrm{Ni}^{2+}$ cation is present) along the molecular backbone.

Another way to quantify the charge transfer (CT) contribution is to consider the unbalance of charge between the two fragments. In fact, if the charge on two isolated components is equal to the charge when they are interacting, the total CT is zero. In the present case, the contribution of the CT, though small, translates into a partial transfer of charge from one fragment to the other. In particular, by means of the Bader charge analysis distribution, we calculated the excess (depletion) of charge at the interface as:

$$
\Delta \rho(z)=\rho_{\mathrm{G} / \mathrm{SAM}}(z)-\left[\rho_{\mathrm{G}}(z)+\rho_{\mathrm{SAM}}(z)\right]
$$

were $\rho_{\mathrm{G} / \mathrm{SAM}}$ is the charge density of the full system and $\rho_{\mathrm{G}}$ and $\rho_{\text {SAM }}$ are the charge densities on the two non-interacting fragments. 

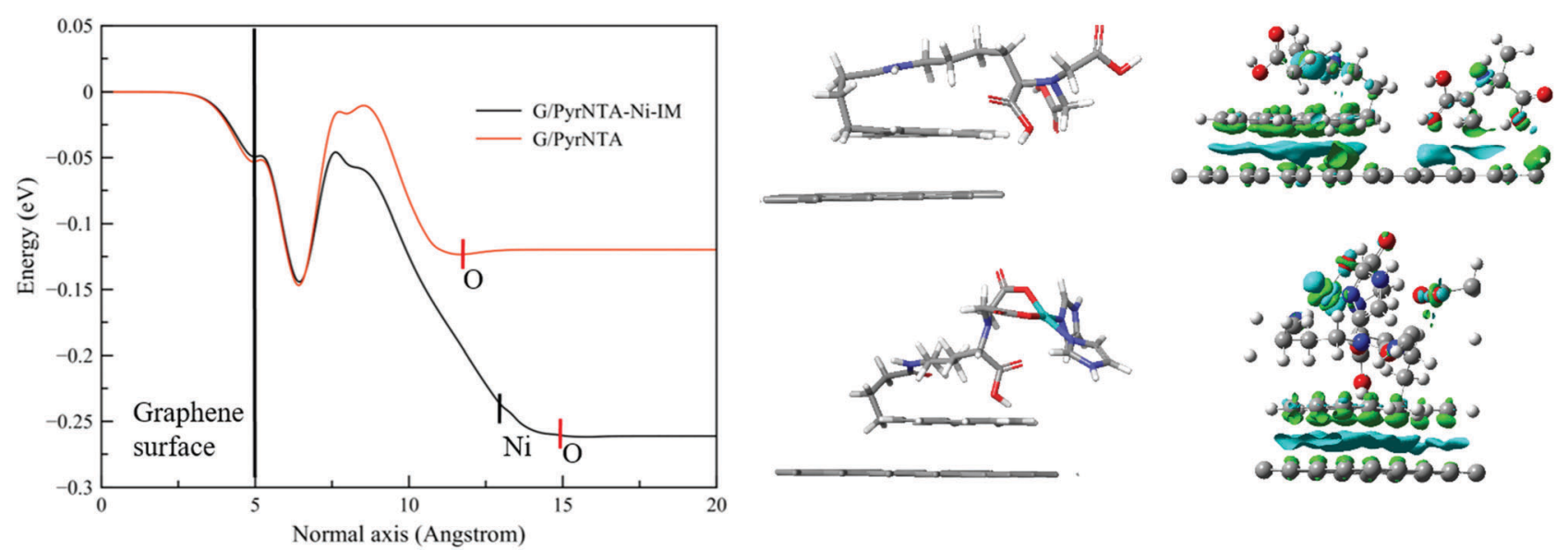

Fig. 3 Evolution of the charge transfer contribution to the work function. From left to right we move away from the graphene surface (indicated as a black bar) towards the SAM layer. For both systems investigated, the $\Delta \Phi_{\mathrm{CT}}$ contribution increases up to the end of the molecular backbone. The positions of the last oxygen atoms (the furthest atom from graphene) and the $\mathrm{Ni}^{2+}$ cation are shown. The central panel shows the optimized structures of the G/pyrNTA (top) and G/pyrNTA-Ni-IM (bottom). The right inset shows the difference in charge density after physisorption for G/pyrNTA (top) and G/pyrNTA-Ni-IM (bottom). Grey colour refers to carbon, red to oxygen, blue to nitrogen, white to hydrogen and cyan to nickel atoms.

We found a negligible excess of electrons of $-0.004|\mathrm{e}|$ on the SAM for the G/pyrNTA interface, while for the system with the $\mathrm{Ni}^{2+}$ cation and the two imidazole molecules this value is increased almost 10-fold to $0.02|\mathrm{e}|$. Interestingly, when the chelation of $\mathrm{Ni}^{2+}$ was considered, the flow of charge was reversed, going from the SAM to graphene, in a metal-to-ligand charge transfer (MLCT) process. As a result, we can speculate that the positive shift of the work function for both systems arises from the different dipole moments present on the two structures, with a negligible CT contribution for the G/pyrNTA interface, resulting in an electron flow from graphene to the SAM. On the other hand, for the G/pyrNTA-Ni-IM interface this contribution is larger, with electron flow from the SAM to the graphene.

To confirm the possibility of a MLCT process, analysis of the density of states (DOS) was performed. Fig. 4 shows the total DOS for the G/SAM interface and their projection on the two fragments, graphene and SAM as well as the projected DOS (PDOS) over each atom type, for the G/pyrNTA interface.

For this system, the state responsible for the Highest Occupied Crystal Orbital (HOCO) is pinned to the Fermi level of graphene, at $-3.70 \mathrm{eV}$ and has a mixed contribution from both the graphene and the SAM molecule, while for the Lowest Unoccupied Crystal Orbital (LUCO) there is a stronger contribution from the graphene surface. This result is more evident when the atomic PDOS are considered, for which the HOCO peak is a sum of the different contributions from carbon and nitrogen atoms, while the LUCO is purely constituted of carbon DOS. Additionally, the shape of the HOCO/LUCO orbitals (depicted in Fig. 4) confirms again the localization of the HOCO on the pyrene part of the SAM and on the NTA moiety, with the LUCO being more delocalized over both the graphene and pyrene components. This delocalization of the LUCO is the main phenomenon responsible for the negligible CT observed for this interface, from which the major molecular backbone contribution is responsible for the positive shift in the work function.
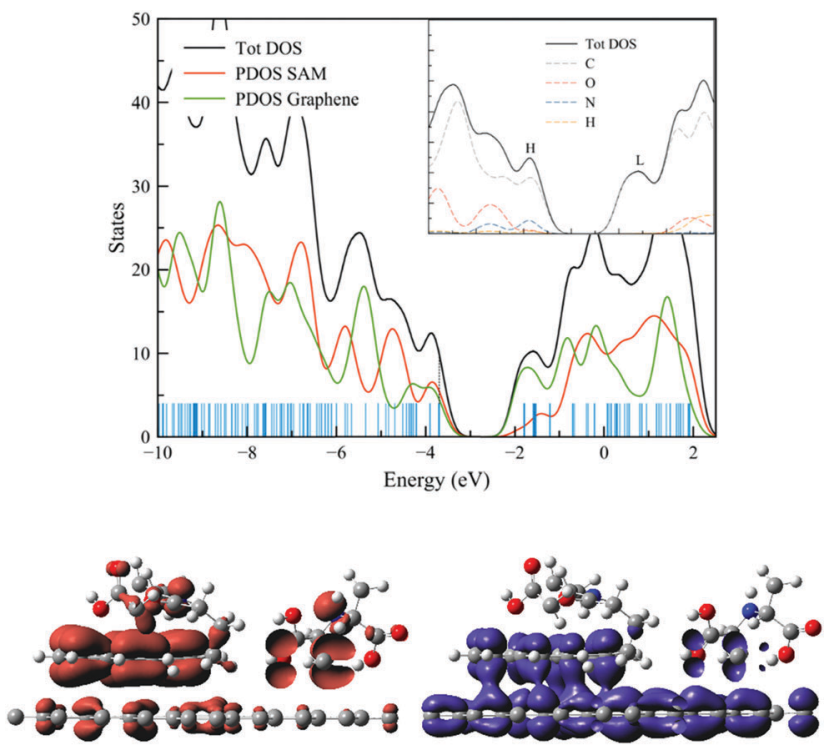

Fig. 4 Total DOS and that projected over the fragments and over each atom (top) for the G/pyrNTA interface. The vertical blue lines indicate the eigenvalues; the dotted black line indicates the position of the Fermi energy level. The peaks corresponding to the Highest Occupied Crystal Orbital (HOCO) and the Lowest Unoccupied Crystal Orbital (LUCO) levels are also indicated. The shapes of the HOCO and LUCO are depicted in red and blue, respectively.

A different scenario is realized when the G/pyrNTA-Ni-IM interface is considered (Fig. 5). As previously, the HOCO is pinned to the Fermi energy of graphene, at $-3.30 \mathrm{eV}$, but now the contribution for the HOCO peak is due to the SAM only, while for the LUCO the main contribution comes from graphene, with a minor contribution from the SAM. The analysis of the atomic PDOS reveals that the $\mathrm{Ni}^{2+}$ cation is the main element responsible for the HOCO peak, while, as expected, only carbon is responsible for the LUCO. 

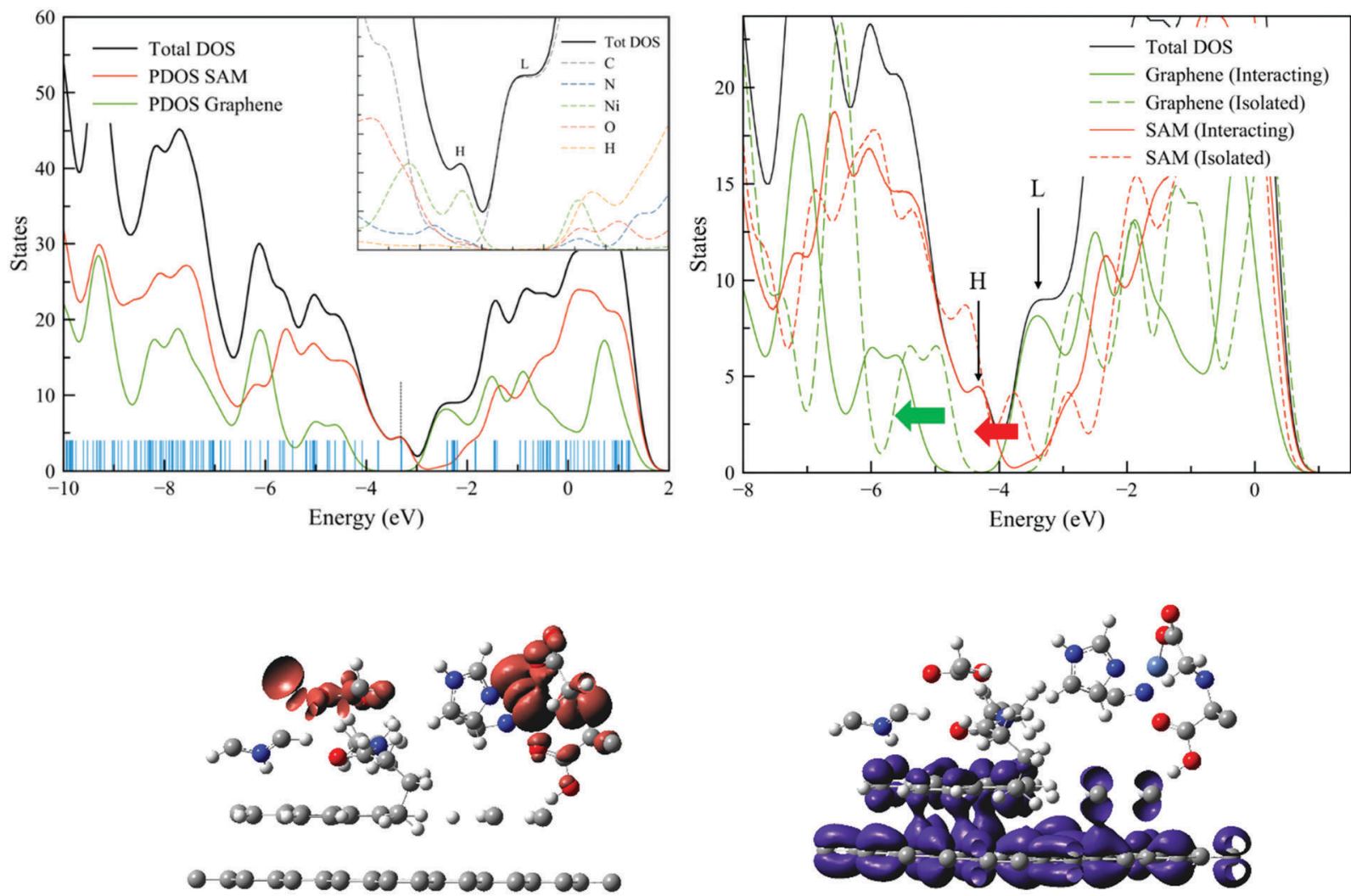

Fig. 5 Total and projected DOS over the fragments and atoms (left); DOS relative shift due to SAM-G interaction (right) for the G/pyrNTA-Ni-IM interface. The vertical blue lines indicate the eigenvalues; the dotted black line indicates the position of the Fermi energy level. The peaks corresponding to the Highest Occupied Crystal Orbital (HOCO) and the Lowest Unoccupied Crystal Orbital (LUCO) levels are also indicated. The shapes of the HOCO and LUCO are depicted in red and blue, respectively. The red and green arrows indicate the downshift of the DOS due to the G/SAM interaction.

As previously shown, this result is corroborated by the orbital shapes, confirming the sole contribution of the SAM to the HOCO, which is strongly localized on the bonding ' $\mathrm{d}$ orbital' of $\mathrm{Ni}^{2+}$, while the LUCO represents a delocalization over the graphene layer and the pyrene moiety of the SAM. This last DOS analysis confirms the flow of charge from the SAM to the graphene in a MLCT process, as observed in the charge analysis. Moreover, when the DOS of the interacting and non-interacting components of the surface are analysed, the $0.02 \mathrm{eV}$ CT contribution is restored. In fact, compared to the isolated case (i.e. SAM and graphene not interacting), the DOS of the interacting SAM are downshifted by $0.58 \mathrm{eV}$, while for graphene the shift is of $0.60 \mathrm{eV}$. This small difference in the energy shift of $0.02 \mathrm{eV}$ is the driving force for the electron flow generated at the interface. In conclusion, the creation of the G/pyrNTA-Ni-IM interface leads to a strong positive shift of the work function of graphene mainly due to the molecular backbone of the SAM, but with a non-negligible CT contribution arising from the mismatch of $0.02 \mathrm{eV}$ in the energy level alignment of the two components of the interface which translates into an overall electron flow from the SAM to graphene.

\section{Elemental and electrochemical analysis of the graphene-pyrene-NTA electrodes}

In order to experimentally investigate the electron transfer properties of the systems modelled by the theoretical calculations, we prepared the respective electrodes on an FTO/SLG substrate, then analysed them elementally by energy-dispersive X-ray spectroscopy (EDX) to confirm the presence and assess the coverage of SLG with pyrNTA-Ni SAM. The EDX mapping image and a quantitative elemental analysis (see Fig. 6) demonstrate the regular distribution of the $\mathrm{N}$ and $\mathrm{Ni}$ atoms $(0.90 \% \mathrm{~N}$ and $0.15 \% \mathrm{Ni}$ ) on the electrode surface. These results reveal at an atomic scale that $\mathrm{N}$ and $\mathrm{Ni}$ atoms doped the SLG surface.

To assess the electron transfer properties of the FTO/SLG electrodes functionalised with the SAM, we characterised them by photochronoamperometry at various potentials. We compared the photocurrent densities obtained from three FTO-based electrodes modified with the pyrene-nitrilotriacetic acid moiety self-assembled on graphene (G/pyrNTA), the graphene electrode containing the pyrene-NTA group coordinated with $\mathrm{Ni}^{2+}$ cations (G/pyrNTA-Ni) and the graphene/pyrene-NTA-Ni layer fully coordinated with imidazole (G/pyrNTA-Ni-IM). As a control, the FTO/ SLG electrode was used (see Fig. 7).

The chronoamperometric analysis indicates the highest photocurrent generation of $14.9 \mathrm{nA} \mathrm{cm}^{-2}$ on the G/pyrNTA sample when no external bias is applied (Fig. 7), with a decrease in current going from G/pyrNTA-Ni $\left(10.0 \mathrm{nA} \mathrm{cm}^{-2}\right)$ to G/pyrNTA$\mathrm{Ni}-\mathrm{IM}\left(3.4 \mathrm{nA} \mathrm{cm}^{-2}\right)$, in agreement with our theoretical calculations which provided the mode of the preferential electron transfer from graphene to the pyrNTA SAM for the first and the 

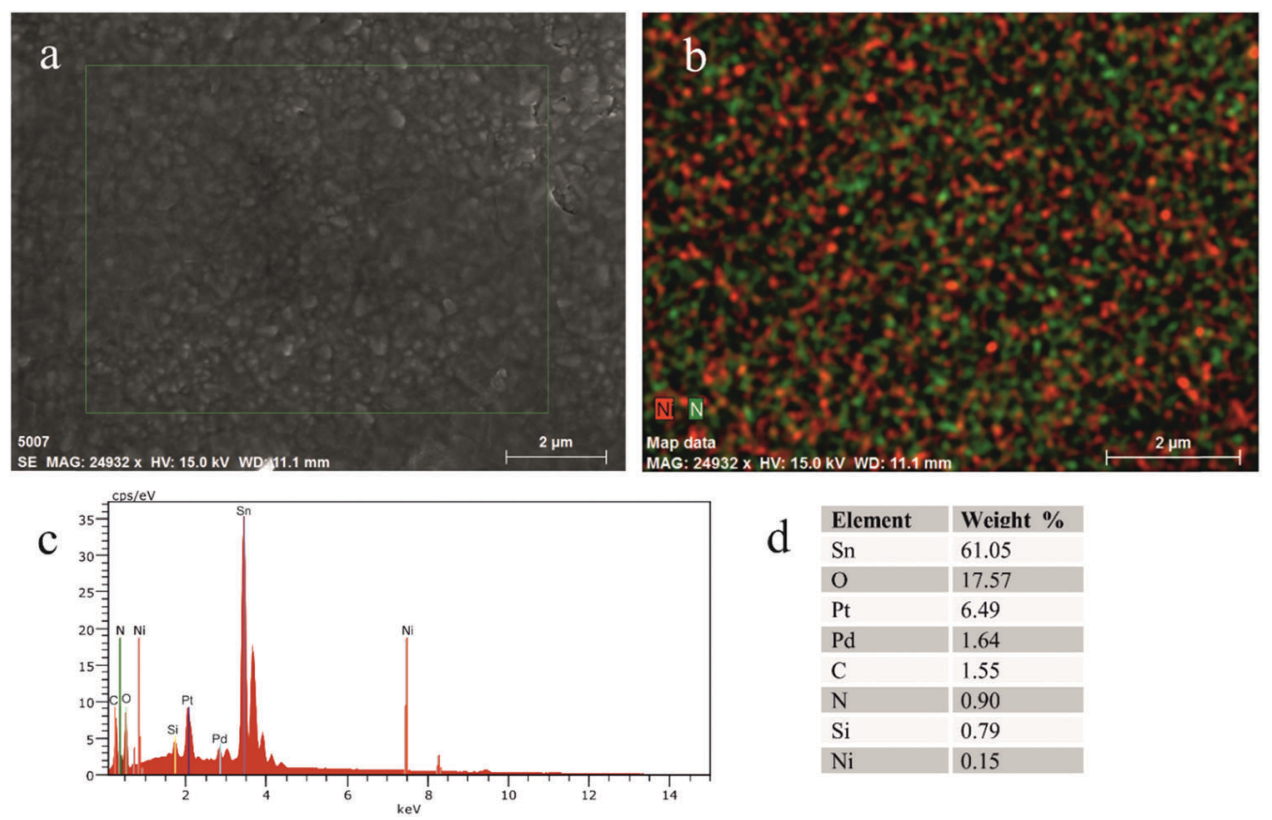

Fig. 6 Elemental analysis of the SAM-modified SLG surface. (a) FE-SEM images of the Ni/NTA-pyrene/SLG/FTO surface. (b) EDX mapping of the Ni and $\mathrm{N}$ atoms taken from the selected area of the SEM image. The red and green spots in (b) represent the Ni and N atoms, respectively. (c) EDX spectrum and (d) quantitative elemental analysis of the selected area (boxed in a) of the EDX map.

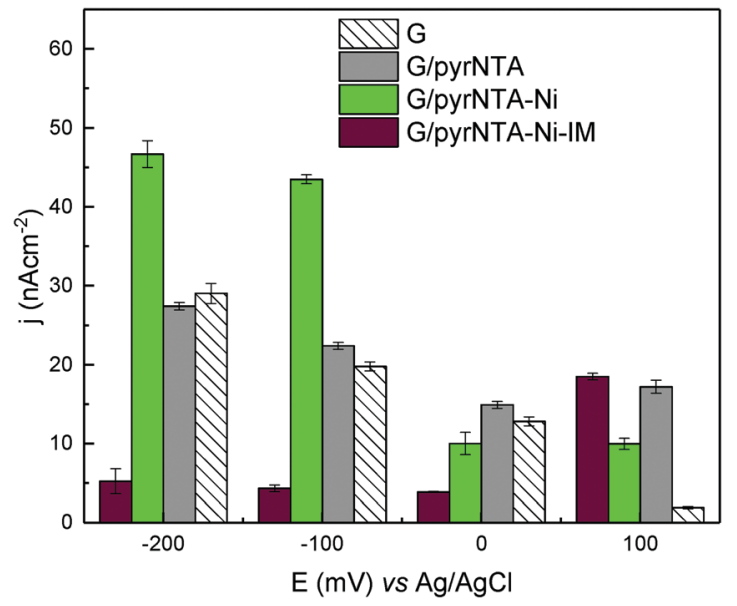

Fig. 7 Photocurrent density generation at the respective SAM films at different applied potentials vs. Ag/AgCl. The current density values constitute the average value from at least two measurements with the error bars representing the standard deviation between those measurements $(n=2)$.

opposite CT, from SAM to graphene, when $\mathrm{Ni}^{2+}$ and imidazole are present (see Fig. 3). We observed a 2 -fold increase of current for the G/pyrNTA-Ni type of assembly (up to $46.7 \mathrm{nA} \mathrm{cm}{ }^{-2}$ ) when applying a negative potential of either -100 or $-200 \mathrm{mV}$, compared to the G/pyrNTA system. As expected, with no bias applied, the current densities were much smaller, in the range of 7-14 nA cm${ }^{-2}$, confirming our theoretical calculations showing the positive effect of $\mathrm{Ni}^{2+}$ cations on the CT from the pyrene SAM to graphene. Introduction of imidazole to the G/pyrNTA-Ni layer caused a significant decrease in the photocurrent generation with negative potential or no bias applied (see Fig. 7). A 10-fold photocurrent reduction was observed for the G/pyrNTA-Ni-IM sample compared to the G/pyrNTA-Ni assembly, in line with our theoretical calculations. Moreover, the current densities recorded from G/pyrNTA-Ni-IM assemblies at no bias were even smaller compared with the graphene monolayer alone, suggesting a dominating effect of charge recombination at the interface with the introduction of imidazole, due to the opposite flow of charges from SAM to graphene.

On the other hand, upon application of a positive potential of $+100 \mathrm{mV}$, the anodic photocurrents were significantly increased in the presence of imidazole up to $18.5 \mathrm{nA} \mathrm{cm}-2$ (compare the photocurrent densities from the G/pyrNTA-Ni-IM and G/pyrNTA-Ni assemblies in Fig. 7), confirming our theoretical predictions of the preferential electron flow from SAM to graphene.

The predicted theoretical behaviour of the SAM on graphene is thus confirmed by photoelectrochemical characterisation. A direct comparison can be made for the situation without bias, in which the electrons flow from the graphene monolayer to the SAM (G/pyrNTA), and a reduction in charge flow is observed when the metal centre and imidazole are present, since the flow of electrons is reversed, going now from the SAM to graphene (G/pyrNTA-Ni-IM). Moreover, these finding are strengthened when a bias is applied; in fact, with a cathodic current the flow of electrons is decreased when G/pyrNTA-Ni-IM is considered with respect to G/pyrNTA, since the external field has the opposite direction to the electron flow, acting to increase the electron injection barrier. On the other hand, when an anodic current is applied, the electron flow is enhanced, since now both the external field and the charge flow are oriented in the 
same direction, with the external field acting to decrease the electron injection barrier.

\section{Conclusions}

Here, we report the quantum mechanical and photoelectrochemical characterisation of graphene modified with a pyrenenitrilotriacetic acid self-assembled monolayer within the confines of the electrochemical investigation of charge transfer between the respective components. Both theoretical calculations and photochronoamperometric analysis indicated an increase of electron transfer within the G/SAM samples by the introduction of the $\mathrm{Ni}^{2+}$ cations, with an electron flow from the SAM to the graphene monolayer. The calculated work function shift is enhanced from 0.42 to $1.29 \mathrm{eV}$ on going from G/pyrNTA to G/pyrNTA-Ni-IM systems, respectively. Moreover, the main contribution to this shift has been found to arise from the molecular backbone rather than the charge transfer, which is similar for both systems, with values between -0.1 and $-0.3 \mathrm{eV}$. Interestingly, the metal centre plays a crucial role in the charge flow direction. In fact, for the G/pyrNTA system the electrons flow from graphene to the SAM, while for the G/pyrNTA-Ni-IM system the flow is reversed, going from the SAM to graphene. These data have been validated by an in-depth analysis of the density of states (DOS) of the two systems, which elucidates the effect of the interaction between graphene and the SAM, and the orbitals responsible for the charge transfer at the interface. These theoretical findings are in full agreement with photoelectrochemical data showing that the presence of $\mathrm{Ni}^{2+}$ cations coordinated with imidazole decreases the electron flow around 4-fold and 3-fold compared to the pyrNTA and graphene alone samples, when no bias is applied. In addition, when an anodic current is generated the DET is enhanced since the electron injection barrier is decreased. In contrast, with the generation of the cathodic current the injection barrier for electrons is increased, leading to an overall decrease of DET. This full characterization of the G/SAM interface is a first step towards the assembly of more complex and challenging systems in which biological components are added, to form a fully functioning bio-organic nanodevice.

\section{Author contribution}

SO and BT performed the quantum calculations. MK performed the electrode functionalisation and electrochemical characterisation. KO, EH and GU constructed the SLG/FTO electrodes and performed the materials' characterisation. SO and JK conceived the study, analysed the data and co-wrote the manuscript.

\section{Conflicts of interest}

There are no conflicts to declare.

\section{Acknowledgements}

SO acknowledges financial support from the Polish National Science Centre, grant UMO-2015/19/P/ST4/03636 (POLONEZ 1) for the funding from the European Union's Horizon 2020 research and innovation program under the Marie SkłodowskaCurie grant agreement No. 665778. JK and MK acknowledge the financial support from the Polish National Centre for Research and Development (grant no. DZP/POLTUR-1/50/2016, agreement no. 5/POLTUR-1/2016 to JK). EA, CGU and KO acknowledge financial support from the Scientific and Technological Research Council of Turkey, TUBITAK (Grant: 215M389) in the framework of the bilateral project between the national funding agencies of Turkey and Poland. The calculations were partially performed at the Interdisciplinary Centre for Mathematical and Computational Modelling (ICM, University of Warsaw) under the GA-69-26 computational grant.

\section{References}

1 A. Karimi, A. Othman, A. Uzunoglu, L. Stanciu and S. Andreescu, Nanoscale, 2015, 7, 6909.

2 A. A. Babadi, S. Bagheri and S. B. A. Hamid, Biosens. Bioelectron., 2016, 79, 850.

3 T. Nöll and G. Nöll, Chem. Soc. Rev., 2011, 40, 3564.

4 C. Ley, D. Holtmann, K.-M. Mangold and J. Schrader, Colloids Surf., B, 2011, 88, 539.

5 F. Schröper, A. Baumann, A. Offenhäusser and D. Mayer, Chem. Commun., 2010, 46, 5295.

6 E. Mikuła, M. Sulima, I. Marszałek, A. Wysłouch-Cieszyńska, P. Verwilst, W. Dehaen, J. Radecki and H. Radecka, Sensors, 2013, 13, 11586.

7 E. P. Friis, J. E. Andersen, Y. I. Kharkats, A. M. Kuznetsov, R. J. Nichols, J. D. Zhang and J. Ulstrup, Proc. Natl. Acad. Sci. U. S. A., 1999, 96, 1379.

8 E. A. Della Pia, Q. Chi, J. E. Macdonald, J. Ulstrup, D. D. Jones and M. Elliot, Nanoscale, 2012, 4, 7106.

9 L. Banszerus, M. Schmitz, S. Engels, J. Dauber, M. Oellers, F. Haupt, K. Watanabe, T. Taniguchi, B. Beschoten and C. Stampfer, Sci. Adv., 2015, 1, e1500222.

10 A. K. Geim and K. S. Novoselov, Nat. Mater., 2007, 6, 183. 11 D. A. C. Brownson and C. E. Banks, Analyst, 2010, 135, 2768. 12 D. A. C. Brownson, D. K. Kampouris and C. E. Banks, J. Power Sources, 2011, 196, 4873.

13 D. Chen, L. Tang and J. Li, Chem. Soc. Rev., 2010, 39, 3157. 14 S. Alwarappan, A. Erdem, C. Liu and C.-Z. Li, J. Phys. Chem. C, 2009, 113, 8853.

15 S. D. Minteer, P. Atanassov, H. R. Luckarift and G. R. Johnson, Mater. Today, 2012, 15, 166.

16 T. Kuila, S. Bose, P. Khanra, A. K. Mishra, N. H. Kim and J. H. Lee, Biosens. Bioelectron., 2011, 26, 4637.

17 J. Liu, Y. Qiao, C. X. Guo, S. Lim, H. Song and C. M. Li, Bioresour. Technol., 2012, 114, 275.

18 H. Gao and H. Duan, Biosens. Bioelectron., 2015, 65, 404.

19 A. Alshammari, M. G. Posner, A. Upadhyay, F. Marken, S. Bagby and A. Ilie, ACS Appl. Mater. Interfaces, 2016, 8, 21077. 
20 B. Dinesh, V. Mani, R. Saraswathi and S.-M. Chen, RSC Adv., 2014, 4, 28229.

21 K. P. Prasad, Y. Chen and P. Chen, ACS Appl. Mater. Interfaces, 2014, 6, 3387.

22 Y. Song, H. Liu, L. Wan, Y. Wang, H. Hou and L. Wang, Electroanalysis, 2013, 25, 1400.

23 H. N. Ghosh, J. B. Asbury, Y. Weng and T. Lian, J. Phys. Chem. B, 1998, 102, 10208.

24 D. Beljonne, J. Cornil, L. Muccioli, C. Zannoni, J.-L. Brédas and F. Castet, Chem. Mater., 2011, 23, 591.

25 J. Fujisawa and M. Hanaya, J. Phys. Chem. C, 2018, 122, 8.

26 M. Singh, M. Holzinger, M. Tabrizian, S. Winters, N. C. Berner, S. Cosnier and G. S. Duesberg, J. Am. Chem. Soc., 2015, 137, 2800.

27 K. C. Chandra Bikram, S. K. Das, K. Ohkubo, S. Fukuzumi and F. D'Souza, Chem. Commun., 2012, 48, 11859.

28 P. Garrido-Barros, C. Gimbert-Suriñach, D. Moonshiram, A. Picón, P. Monge, V. S. Batista and A. Llobet, J. Am. Chem. Soc., 2017, 139, 12907.

29 S. C. Feifel, K. R. Stieger, H. Lokstein, H. Lux and F. Lisdat, J. Mater. Chem. A, 2015, 3, 12188.

30 D. Porezag, T. Frauenheim, T. Köhler, G. Seifert and R. Kaschner, Phys. Rev. B: Condens. Matter Mater. Phys., 1995, 51, 12947.

31 M. I. Katsnelson, Graphene: Carbon in Two Dimensions, Cambridge University Press, 2012.

32 S. Yuan, M. Rösner, A. Schulz, T. O. Wehling and M. I. Katsnelson, Phys. Rev. Lett., 2015, 114, 047403.

33 G. Fiori, S. Lebégue, A. Betti, P. Michetti, M. Klintenberg, O. Eriksson and G. Iannaccone, Phys. Rev. B: Condens. Matter Mater. Phys., 2010, 82, 153404.

34 M. Elstner, D. Porezag, G. Jungnickel, J. Elsner, M. Haugk, T. Frauenheim, S. Suhai and G. Seifert, Phys. Rev. B: Condens. Matter Mater. Phys., 1998, 58, 7260.

35 R. Balog, B. Jørgensen, L. Nilsson, M. Andersen, E. Rienks, M. Bianchi, M. Fanetti, E. Laegsgaard, A. Baraldi, S. Lizzit, Z. Sljivancanin, F. Besenbacher, B. Hammer, T. G. Pedersen, P. Hofmann and L. Hornekaer, Nat. Mater., 2010, 9, 315.

36 M. Elstner, P. Hobza, T. Frauenheim, S. Suhai and E. Kaxiras, J. Chem. Phys., 2001, 114, 5149.
37 B. Aradi, B. Hourahine and Th. Frauenheim, J. Phys. Chem. A, 2007, 111, 5678.

38 P. Giannozzi, S. Baroni, N. Bonini, M. Calandra, R. Car, C. Cavazzoni, D. Ceresoli, G. L. Chiarotti, M. Cococcioni, I. Dabo, A. D. Corso, S. de Gironcoli, S. Fabris, G. Fratesi, R. Gebauer, U. Gerstmann, C. Gougoussis, A. Kokalj, M. Lazzeri, L. Martin-Samos, N. Marzari, F. Mauri, R. Mazzarello, S. Paolini, A. Pasquarello, L. Paulatto, C. Sbraccia, S. Scandolo, G. Sclauzero, A. P. Seitsonen, A. Smogunov, P. Umari and R. M. Wentzcovitchet, J. Phys.: Condens. Matter, 2009, 21, 395502.

39 K. Lee, E. D. Murray, L. Kong, B. I. Lundqvist and D. C. Langreth, Phys. Rev. B: Condens. Matter Mater. Phys., 2010, 82, 081101.

40 G. Heimel, I. Salzmann and N. Koch, Chem. Mater., 2011, 23, 359 .

41 D. Chaen and A. Kahn, Adv. Mater., 2003, 15, 271.

42 H. H. Kim, B. Kang, J. W. Suk, N. Li, K. S. Kim, R. S. Ruoff, W. H. Lee and K. Cho, ACS Nano, 2015, 9, 4726.

43 X. Liang, B. A. Sperling, I. Calizo, G. Cheng, C. A. Hacker, Q. Zhang, Y. Obeng, K. Yan, H. Peng, Q. Li, X. Zhu, H. Yuan, A. R. Hight Walker, Z. Liu, L.-M. Peng and C. A. Richter, ACS Nano, 2011, 5, 9144.

44 X. Li, Y. Zhu, W. Cai, M. Borysiak, B. Han, D. Chen, R. D. Piner, L. Colombo and R. S. Ruoff, Nano Lett., 2009, 9, 4359.

45 J. W. Suk, A. Kitt, C. W. Magnuson, Y. Hao, S. Ahmed, J. An, A. K. Swan, B. B. Goldberg and R. S. Ruoff, ACS Nano, 2011, 5, 6916.

46 Y. Chen, X.-L. Gong and J.-G. Gai, Adv. Sci., 2016, 3, 1500343. 47 M. Holzinger, J. Baur, R. Haddad, X. Wang and S. Cosnier, Chem. Commun., 2011, 47, 2450.

48 M. Kondo, K. Iida, T. Dewa, H. Tanaka, T. Ogawa, S. Nagashima, K. V. P. Nagashima, K. Shimada, H. Hashimoto, A. T. Gardiner, R. J. Cogdell and M. Nango, Biomacromolecules, 2012, 13, 432.

49 Y.-J. Yu, Y. Zhao, S. Ryu, L. E. Brus, K. S. Kim and P. Kim, Nano Lett., 2009, 9, 3430. 